\title{
ESE-1 suppresses the growth, invasion and migration of human NSCLC cells and tumor formation in vivo
}

\author{
ZHIYUAN LOU ${ }^{1 *}$, BOK-SOON LEE ${ }^{2 *}$, TAEKYU HA $^{1 *}$, YANRUI XU ${ }^{1}$, \\ HAENG-JUN KIM ${ }^{2}$, CHUL-HO KIM ${ }^{2}$ and SEONG-HO LEE ${ }^{1}$ \\ ${ }^{1}$ Department of Nutrition and Food Science, College of Agriculture and Natural Resources, University of Maryland, \\ College Park, MD 20742, USA; ${ }^{2}$ Department of Otolaryngology, School of Medicine, Ajou University, \\ Yeongtong-Gu, Suwon 16499, Republic of Korea
}

Received January 24, 2018; Accepted July 9, 2018

DOI: $10.3892 /$ or.2018.6560

\begin{abstract}
Lung cancer is the first leading cause of cancer-related death in the United States. Non-small cell lung cancer (NSCLC) is the most common type of lung cancer and is associated with a poor patient prognosis. Identification of promising molecular targets is required for the effective prevention and therapy of NSCLC. Epithelial-specific ETS-1 (ESE-1) belongs to the superfamily of ETS transcription factors. The effect of ESE-1 on tumorigenesis is controversial in several types of cancer while its role in lung cancer remains unknown. The present study was designed to investigate whether ESE-1 expression affects tumorigenic activity using human NSCLC cells and a mouse xenograft model. ESE-1 expression suppressed anchorage-independent growth in soft agar assay and led to an increase in G1 arrest and apoptosis in human NSCLC cells. ESE-1 expression suppressed the invasion and migration of human NSCLC cells. Western blot analysis, RT-PCR and promoter assay indicated that ESE-1 expression was transcriptionally downregulated by treatment of transforming growth factor (TGF)- $\beta$, an EMT (epithelialmesenchymal transition) stimulator. The xenograft study indicated that ESE-1 expression inhibited tumor formation and development. Our data demonstrated that ESE-1 plays a key role as a tumor suppressor in human NSCLC.
\end{abstract}

Correspondence to: DrChul-Ho Kim,Department of Otolaryngology, School of Medicine, Ajou University, 5 Wonchon-Dong, Yeongton-Gu, Suwon 16499, Republic of Korea

E-mail: ostium@ajou.ac.kr

Dr Seong-Ho Lee, Department of Nutrition and Food Science, University of Maryland, 4300 Chapel Lane, College Park, MD 20742, USA

E-mail: slee2000@umd.edu

${ }^{*}$ Contributed equally

Key words: ESE-1, tumorigenesis, EMT, xenograft, NSCLC

\section{Introduction}

Lung cancer is the most life threatening cancer in the world as well as in the United States due to high occurrences of metastasis and drug resistance. In 2017, 2,22,500 Americans were estimated to be diagnosed with lung cancer, and 1,55,870 were estimated to die of lung cancer, accounting for 27 and $25 \%$ in total estimated deaths in males and females, respectively (1). Non-small cell lung cancers (NSCLCs) account for $84 \%$ of all lung cancer cases, and the 5-year survival rate $(15 \%$ for male and $21 \%$ for female) is extremely poor (2). Therefore, identification of promising molecular target is required for the effective prevention and therapy of NSCLCs.

Epithelium-specific ETS-1 (ESE-1) protein, also identified as ELF3 (3), ERT (4), ESX (5) and PDEF (6), belongs to an ETS transcription factor superfamily and is mainly expressed in epithelial-rich tissues including the lung and gut (7). ESE-1 regulates the expression of target genes that determine cancer phenotype including cell division, differentiation and apoptosis (8). However, the effects of ESE-1 on cancer are extremely complex. ESE-1 expression is associated with poor prognosis in colorectal cancer patients (9) while ESE-1 downregulation was found to be related to reduced survival in ovarian cancer (10) and ESE-1 inhibits the invasion of oral squamous cell carcinoma (11). In an in vitro condition, ESE-1 knockdown inhibited cell proliferation in colon cancer cells (9) while ESE-1 was found to facilitate cell growth arrest and apoptosis in the colon cancer cells (12). ESE-1 expression also led to two conflicting results in prostate cancer cells. For example, ESE-1 expression mediated transforming phenotypes (13) while ESE-1 repressed androgen-receptor action and growth/migration of prostate tumors (14). These contradictory results strongly support a dichotomous effect of ESE-1 depending on the cellular context.

In lung tissue, ESE-1 plays a key role in the proliferation and differentiation during airway inflammation and injured airway epithelium repair (15). A high level of ESE-1 expression was detected in the developing fetal and adult lung tissues as well (3). The aim of this study was to investigate whether ESE-1 modulates lung cancer development using human NSCLC cells and a xenograft mouse model. Here, we report that ESE-1 possesses tumor suppressive activity in lung cancer. 


\section{Materials and methods}

Materials. Human NSCLC cell lines (A549, H358, H1299 and H1703) were purchased from the American Type Culture Collection (ATCC; Manassas, VA, USA). TGF- $\beta$ (cat. no. $240-B$ ) was purchased from R\&D System, Inc. (Minneapolis, MN, USA). Antibodies for Smad2 (cat. no. 5339), Smad3 (cat. no. 9513), p-smad2 (cat. no. 8828), p-smad3 (cat. no. 9520), E-cadherin (cat. no. 3195), N-cadherin (cat. no. 4061), Snail (cat. no. 3879), ZO-1 (cat. no. 5406), CDK4 (cat. no. 12790), CDK6 (cat. no. 3136), p27 (cat. no. 3688), MMP-9 (cat. no. 13667), PCNA (cat. no. 2586), cleaved caspase-3 (cat. no. 9661) and $\beta$-actin (cat. no. 5125s) were purchased from Cell Signaling Technology, Inc. (Beverly, MA, USA). Antibodies for ESE-1 (cat. no. SC-376055), VEGF (cat. no. SC-507), Smad4 (cat. no. SC-7966), and siRNA for Smad4 (cat. no. SC-29484) were purchased from Santa Cruz Biotechnology (Dallas, TX, USA). Anti-V5 (cat. no. 46-0708) was purchased from Invitrogen; Thermo Fisher Scientific, Inc. (Waltham, MA, USA). Bcl2 antibody (cat. no. 610538) was purchased from BD Biosciences (San Jose, CA, USA). All chemicals were purchased from Thermo Fisher Scientific or VWR International LLC (Radnor, PA, USA) unless otherwise specified.

Cell culture and establishment of stable cell lines. Human NSCLC cells were grown in DMEM/F12 media supplemented with 10\% fetal bovine serum (FBS). H1299 and H1703 stable cell lines overexpressing LacZ and ESE-1 were generated by transient transfection with expression vectors and selected with $\mathrm{G} 418(800 \mu \mathrm{g} / \mathrm{ml}$ for selection and $400 \mu \mathrm{g} / \mathrm{ml}$ for maintenance).

Cloning of ESE-1 promoters. Human ESE-1 promoter (Reference: NC_018912.2; +1 +713 bp; +1 +1,500 bp) was amplified with primers as indicated in Table I. PCR products were then inserted into the pGL3-basic vector by using In-Fusion cloning method (Clontech Laboratories, Inc., Mountain View, CA, USA). The pGL3-basic vector was linearized with FastDigest HindIII enzyme (Thermo Fisher Scientific, Inc.) and cleaned up with QIAquick PCR purification kit (Qiagen, Inc., Germantown, MD, USA). Internal deletion and point mutation constructs (both single and double) were cloned using the QuickChange Mutagenesis kit (Thermo Fisher Scientific, Inc.) as previously described (16).

Transfection and reporter gene assay. ESE-1 promoters were transiently transfected using PolyJet ${ }^{\mathrm{TM}}$ DNA transfection reagent (SignaGen Laboratories, Rockville, MD, USA) according to the manufacturer's instructions. The cells were harvested in $1 \mathrm{X}$ luciferase lysis buffer. The luciferase activity was measured and normalized to the pRL-null luciferase activity using a Dual-Luciferase assay kit (Promega, Madison, WI, USA) as previously described (16).

SDS-PAGE and western blot analysis. SDS-PAGE and western blot analysis were performed as we previously described (16). The cell lysates were extracted using RIPA buffer and $25 \mu \mathrm{g}$ of protein was loaded onto SDS-polyacrylamide gel (8-12\% depending on molecular size of each protein). After transferring onto a nylon membrane, the extracted proteins were incubated with the primary antibodies $(1: 1,000)$. The antibody information was described in Materials and methods. Chemiluminescence was detected with Pierce ECL Western blotting substrate (Thermo Fisher Scientific, Inc.) and visualized using the ChemiDoc MP Imaging system (Bio-Rad Laboratories, Hercules, CA, USA).

Semi-quantitative RT PCR. Semi-quantitative RT-PCR was performed as we previously described (16). The primer sequences for PCR are as follows: ESE-1, forward 5'-ttagcaac tacttcagtgcgatgt-3' and reverse 5'-gttcttctccacttggtagctgat-3'; Snail, forward 5'-CCTCCCTGTCAGATGAGGAC-3' and reverse 5'-CCAGGCTGAGGTATTCCTTG-3'; GAPDH, forward 5'-GGGCTGCTTTTAACTCTGGT-3' and reverse 5'-TGGCAGGTTTTTCTAGACGG-3'.

Soft agar assay. The bottom layer containing 1\% agar and culture medium was prepared in the 6-well culture plate. A mixture of agar and cells $(5,000$ cells/well) were placed, solidified and incubated in a $37^{\circ} \mathrm{C}$ humidified $\mathrm{CO}_{2}$ incubator. Then, a layer of growth medium $(200 \mu \mathrm{l})$ was added over the upper layer of agar to prevent desiccation every 2 days. Cells were grown for 2 weeks and stained overnight with nitro-blue tetrazolium chloride solution at $37^{\circ} \mathrm{C}$. Images were captured by ChemiDoc MP Imaging system (Bio-Rad Laboratories).

Invasion assay. Cell invasion was analyzed using an invasion assay kit (Corning Inc., Corning, NY, USA) according to the manufacturer's instructions. Briefly, cells were suspended and plated in an upper invasion chamber (24-wells) of a Transwell with Matrigel. The lower chamber was added with $10 \%$ FBS medium. After $12 \mathrm{~h}$ of incubation, the cells in the chamber were removed with a cotton swab. After staining, images were captured under an inverted microscope (Nikon Eclipse Ti; Nikon Corp., Tokyo, Japan).

Scratch wound assays. The cells $\left(5 \times 10^{4}\right.$ cells/well) were seeded onto 6-well tissue culture plates and grown overnight. A linear wound was generated using a sterile $20-\mu 1$ pipette tip. After $22 \mathrm{~h}$, images were captured by an inverted microscope (Nikon Eclipse Ti; Nikon).

Cell cycle distribution and analysis of apoptosis. Cell cycle analysis was performed as we previously described (17). The cells were collected through typsin-EDTA treatment and stained with propidium iodide (PI). After washing, the cell cycle distribution was analyzed using FACS flow cytometry at the University of Maryland MPRI Flow Cytometry and Cell Sorting Facility. Apoptosis was measured using Cell Death Detection ELISA (Roche Diagnostics, Indianapolis, IN, USA) according to the manufacturer's instructions.

Xenograft study. The animal study was approved by the Animal Care and Use Committee (Ajou University, Suwon, Korea). Fourteen male BALB/c nude mice aged 6 weeks with average weight of $25 \mathrm{~g}$ were purchased from Harlan Laboratories, Ltd., (Bicester, UK) and maintained ad libitum at $23^{\circ} \mathrm{C}$ temperature and 12-h light/dark cycle. The mice were injected s.c. in the left flank with H1299 stable cell lines overexpressing LacZ 
Table I. Sequences of primers used for cloning of the ESE-1 promoters.

Primer name Sequence

ESE1-promoter-713-F
ESE1-promoter-713-R
ESE1-promoter-1500-F
ESE1-promoter-1500-R
ESE1-promoter-1500- $\Delta \mathrm{D}-\mathrm{F}$
ESE1-promoter-1500- $\Delta \mathrm{D}-\mathrm{R}$
ESE1-promoter-1500- $\Delta \mathrm{P}-\mathrm{F}$
ESE1-promoter-1500- $\Delta \mathrm{P}-\mathrm{R}$
ESE1-promoter-1500- $\triangle \mathrm{D} \& \mathrm{P}-\mathrm{F}$
ESE1-promoter-1500- $\triangle \mathrm{D} \& \mathrm{P}-\mathrm{R}$
ESE1-promoter-1500-mD-F
ESE1-promoter-1500-mD-R
ESE1-promoter-1500-mP-F
ESE1-promoter-1500-mP-R
ESE1-promoter-1500-mD\&P-F
ESE1-promoter-1500-mD\&P-R

5'-CGATCTAAGTAAGCTTGGGAAGAACTTTCAAGCAGAG-3'
5'-CCGGAATGCCAAGCTTGGCTTTATAGTGTGTCCCCTG-3'
5'-CGATCTAAGTAAGCTTCTGGGCGACAGAGCGAGAC-3'
5'-CCGGAATGCCAAGCTTGGCTTTATAGTGTGTCCCCTG-3'
5'-GGAAGAGATCTTAGGGATTATTACCTCATTTTTATAG-3'
5'-GTTTCCCCATCTATAAAAAATGAGGTAATAATCCCTAAG-3'
5'-CGGGCTGAGTCCATAGAGACATGGGGGACTC-3'
5'-CCCTGTGGGCAGAGTCCCCCATGTCTCTATGG-3'
5'-GGAAGAGATCTTAGGGATTATTACCTCATTTTTTATAG-3'
5'-GTTTCCCCATCTATAAAAAATGAGGTAATAATCCCTAAG-3'
5'-GGGATTATTAAATTTTTTCAACCTCATTTTTTATAG-3'
5'-CTATAAAAAATGAGGTTGAAAAAATTTAATAATCCC-3'
5'-GAGACAACCATTTTCATGGGGGAC-3'
5'-GTCCCCCATGAAAATGGTTGTCTC-3'
5'-GGGATTATTAAATTTTTTCAACCTCATTTTTTATAG-3'
5'-CTATAAAAAATGAGGTTGAAAAAATTTAATAATCCC-3'

F, forward; R, reverse; $\mathrm{D}$, distal; P, proximal; $\Delta$, internal deletion; m, point mutation.

and ESE-1 (5x10 6 cells mixed with Matrigel, 1:1). After cell injection, the tumor formation was monitored twice a week and tumor size was measured with a caliper (Mitutoyo, Utsunomiya, Japan) and the volumes were estimated using the formula: $a \times b^{2} \times 0.52$, where ' $a$ ' is the longest and ' $b$ ' is the shortest diameter. Thirty five days after injection, the mice were sacrificed by carbon dioxide asphyxiation and the tumors were harvested and subjected to immunohistochemistry and western blot analysis.

Immunohistochemistry. Immunohistochemistry was performed to analyze the expression of ESE-1, PCNA and cleaved caspase-3. Paraffin tissue sections ( $4 \mu \mathrm{m}$ thick) were briefly deparaffinized with xylene and rehydrated through alcohol, and washed in distilled water. After $\mathrm{H}_{2} \mathrm{O}_{2}$-induced inactivation for endogenous peroxidase activity and antigen retrieval in pepsin (Dako, Carpinteria, CA, USA) at $37^{\circ} \mathrm{C}$ for $30 \mathrm{~min}$, the tissue sections were incubated with primary antibodies for ESE-1 (1:100), PCNA $(1: 4,000)$ and cleaved caspase-3 $(1: 2,000)$ overnight at $4^{\circ} \mathrm{C}$. After washing, signals were detected with 3,3-diaminobenzidine tetrahydrochloride (DAB) and the sections were counterstained with hematoxylin.

Statistical analysis. Statistical analysis was performed by Student's t-test. Data represent mean \pm SD from three replicates.

\section{Results}

Basal expression of ESE-1 in human NSCLC cells. First, we determined the basal expression of ESE-1 in four different human NSCLC cell lines (A549, H358, H1299 and H1703). As shown in Fig. 1A, A549 and H358 were ESE-1 abundant whereas H1299 and H1703 expressed low levels of ESE-1. We generated H1299 and H1703 stable cell lines overexpressing LacZ (control) and ESE-1 by transfecting V5-tag-LacZ (control) and the V5-tag-ESE-1 expression vector into these cell lines and subsequent selection with G418. Overexpression of LacZ and ESE-1 were validated through V5 expression as well as endogenous ESE-1 expression (Fig. 1B.)

ESE-1 suppresses anchorage-independent growth of human NSCLC cells. Next, we performed soft agar assay to ascertain whether ESE-1 overexpression affects the anchorage-independent growth of H1299 and H1703 stable cell lines. As shown in Fig. 1C, the number of colonies was 3-fold higher in the LacZ-expressing H1299 cells compared to the number of colonies noted in the ESE-1-expressing H1299 cells. Similar result was obtained from ESE-1-overexpressing H1703 cells. The result indicates that ESE-1 possesses anti-growth activity in human NSCLC cells.

'To investigate whether retarded growth by ESE-1 overexpression is associated with cell growth arrest and apoptosis, cell cycle distribution and the fraction of apoptotic cells were compared between LacZ- and ESE-1-expressing H1299 stable cells. Cell cycle analysis data showed that ESE-1 overexpression resulted in a significant increased percentage of cells in the G1 phase (LacZ, 27.7 \pm 0.5 vs. ESE-1, 33.3 $\pm 1.3 \%$ ) and a decrease in the $S$ phase (LacZ, 57.2 \pm 1.7 vs. ESE-1, $48.6 \pm 2.0 \%$ ), and no significant change in the $\mathrm{G} 2$ phase (LacZ, $15.0 \pm 1.2$ vs. ESE-1, 18.1 $\pm 2.4 \%$ ) (Fig. 1D). In addition, the number of apoptotic cells was increased by 1.7 -fold in the ESE-1-expressing H1299 cells compared to that noted in the control cells (Fig. 1E). We also measured the expression of several cell cycle- and apoptosis-regulating marker proteins using western blot analysis. As shown in Fig. 1F, ESE-1 expression led to a decrease in CDK4 and CDK6 and an increase in 
A

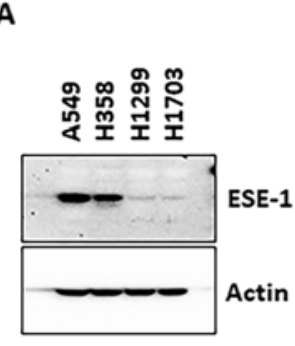

B

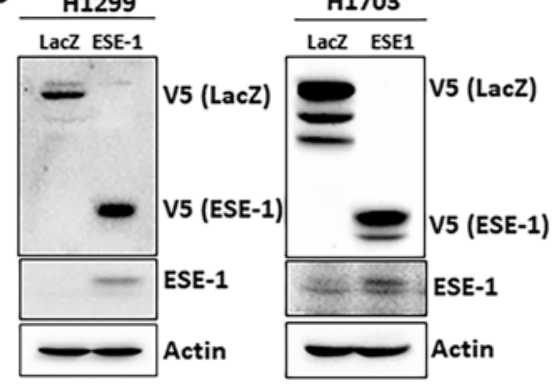

C

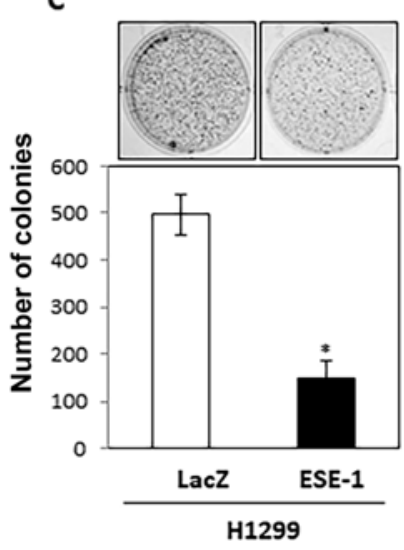

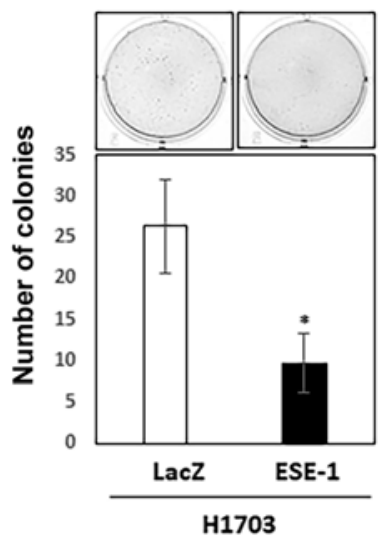

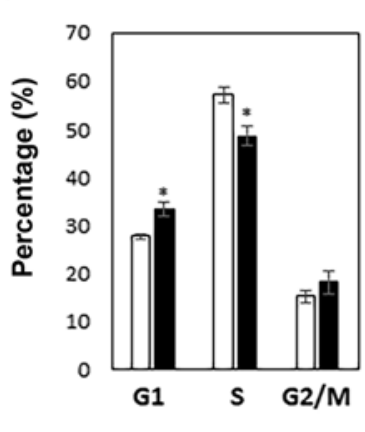

E

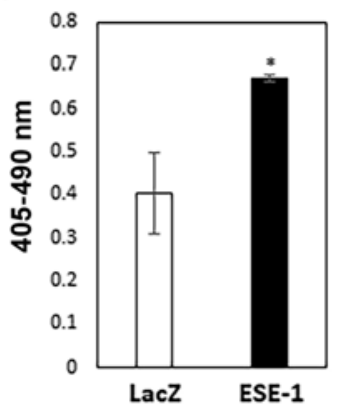

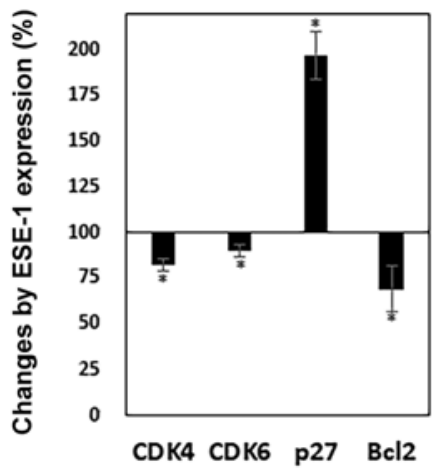

Figure 1. ESE-1 suppresses anchorage-independent growth of human NSCLC cells. (A) Cell lysates obtained from A549, H358, H1299 and H1703 cells were subjected to western blot analysis for ESE-1 and actin. (B) H1299 and H1703 cells were transfected with V5-tag-LacZ (control) and V5-tag-ESE-1 expression vectors and selected with G418. The cell lysates were subjected to Western blot analysis for V5 and ESE-1 for validation of stable cells. (C) Soft agar assay was performed as described in the Materials and methods section. The images were captured 2 weeks after inoculation. (D) Cell cycle distribution was performed by staining the cells with propidium iodide and subsequent FACS analysis. (E) Apoptosis was measured using Cell Death Detection ELISA. (F) The cell lysates were subjected to western blot analysis for CDK4, CDK6, p27, Bc12 and actin (left). Data represent the mean \pm SD from three independent experiments (right). ${ }^{*} \mathrm{P}<0.05$ vs. the control. NSCLC, non-small cell lung cancer; ESE-1, epithelial-specific ETS-1.

p27. We also found that ESE-1 overexpression decreased Bcl2 expression.

ESE-1 suppresses invasion and migration of human NSCLC cells. Based on our finding that ESE-1 possesses tumor-suppressive activity, we further explored whether ESE-1 possesses anti-metastatic activity by performing in vitro cell invasion and migration assays. As indicated in Fig. 2A, the wound healing assay indicated that ESE-1-expressing cells had reduced migration capacity than that of the control cells. In addition, the ESE-1-expressing stable cell line showed lower invasive ability when compared to the control cells (Fig. 2B). These results imply that ESE-1 overexpression inhibits the metastatic activity of human NSCLC cells in vitro. Western blot analysis showed that expression of MMP-9 was decreased in the ESE-1-expressing cells compared to that noted in the control cells (Fig. 2C). An angiogenic protein (VEGF) and a tight junction protein (ZO-1) were also downregulated by ESE-1 overexpression (Fig. 2C).

ESE-1 is a target of TGF- $\beta$ in human NSCLC cells. To further study the possible anti-metastatic mechanism mediated by ESE-1, we aimed to ascertain whether epithelial-mesenchymal transition (EMT) influences the expression of ESE-1. Since TGF- $\beta$ is a major trigger of EMT, we treated ESE-1 abundant
A549 and H358 cells with TGF- $\beta$ in a low serum $(0.5 \%)$ condition. Treatment of TGF- $\beta$ to A549 cells led to morphological changes characteristic of EMT (Fig. 3A) which was accompanied by increased phosphorylation of Smad2 and Smad3 (Fig. 3B, top panel). TGF- $\beta$ also decreased the expression of $\mathrm{E}$-cadherin and increased the expression of $\mathrm{N}$-cadherin and Snail in the A549 and H358 cells (Fig. 3B, bottom panels). Notably, TGF- $\beta$ treatment decreased expression of ESE-1 in these two cell lines (Fig. 3B, bottom panels). Treatment of TGF- $\beta$ to A549 cells also showed time-dependent downregulation of ESE-1 protein (Fig. 3C) and ESE-1 mRNA (Fig. 3D). The time-point that ESE-1 began to decrease was earlier $(4 \mathrm{~h})$ than the time point that EMT marker proteins (E-cadherin and $\mathrm{N}$-cadherin) were changed. These data propose a potential link between ESE-1 downregulation and EMT. Finally, we produced two ESE-1 promoter clones spanning to $-1,500 /+1$ and $-713 /+1$ and measured the transcriptional activity (Fig. 3E). The results indicate that treatment of TGF- $\beta$ suppressed luciferase activity by 43.3 and $45.8 \%$ in the $-1,500 /+1$ and $-173 /+1$ clones, respectively. These data indicate that ESE-1 is an inverse downstream target protein of TGF- $\beta$ during EMT.

ESE-1 suppresses formation and development of tumors in vivo. Finally, we verified the anti-cancer activity of ESE-1 
A

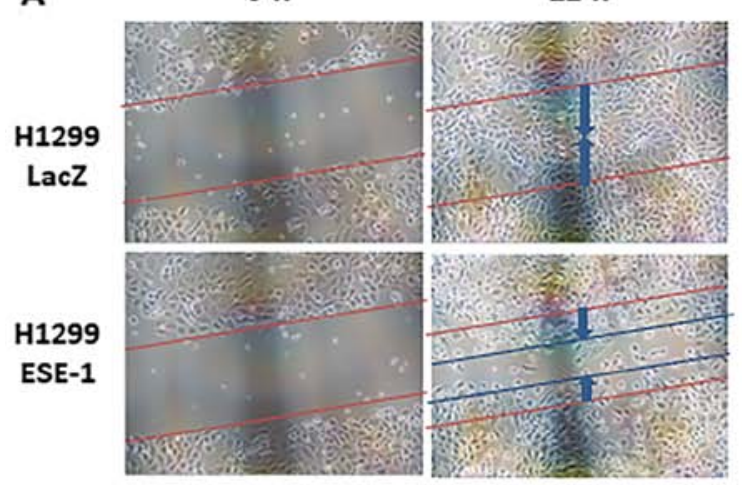

$\mathrm{Oh}$

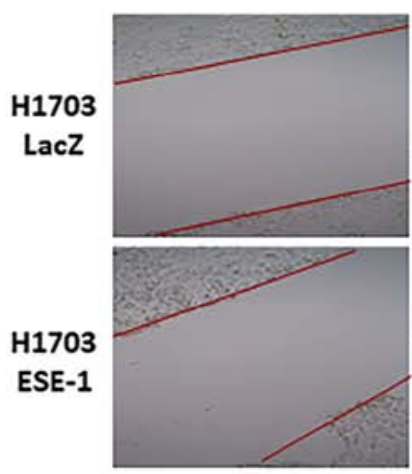

$22 \mathrm{~h}$

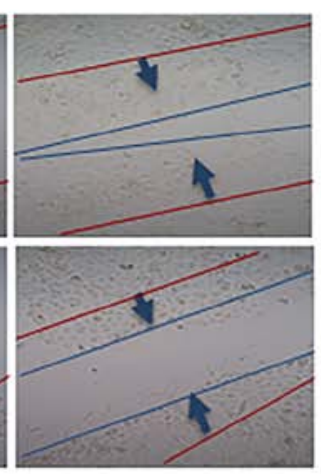

B
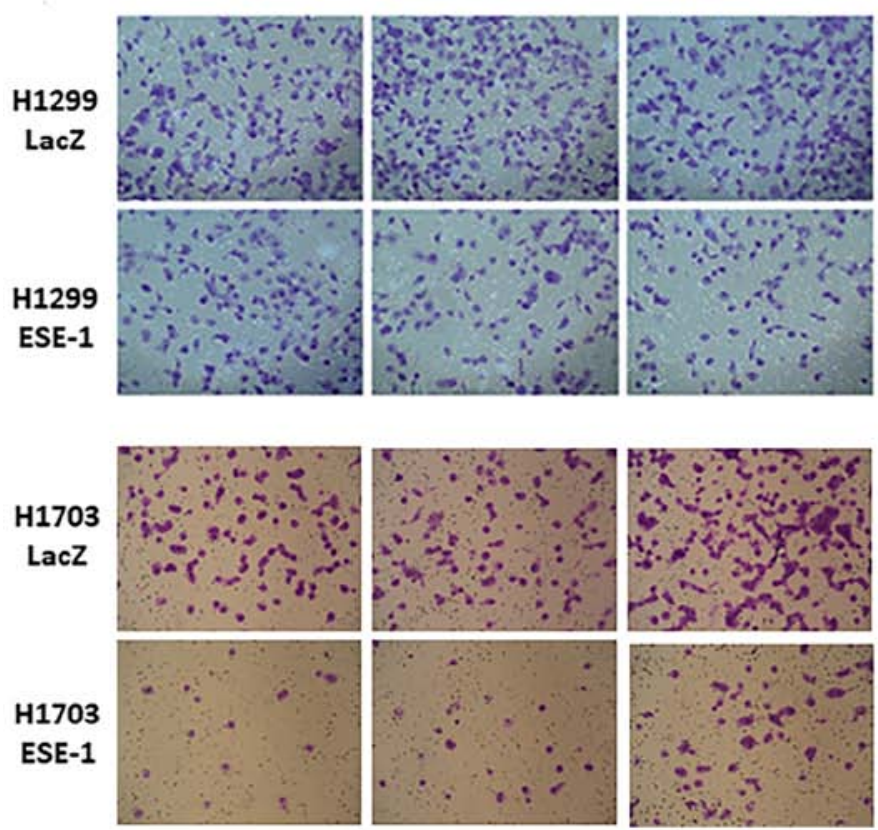

C
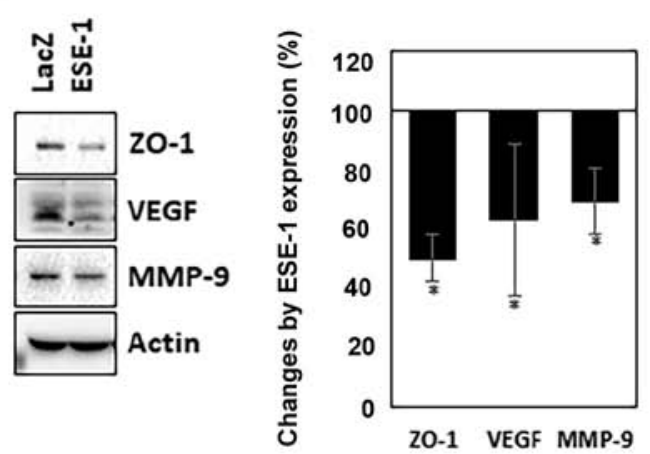

Figure 2. ESE-1 suppresses the invasion and migration of human NSCLC cells. (A) The cells were subjected to wound healing assay for 0 and 22 h. (B) The cells were subjected to Transwell invasion assay. After $12 \mathrm{~h}$ incubation, the cells were stained, and images were captured using a microscope. (C) The cell lysates were subjected to western blot analysis for MMP-9, VEGF, ZO-1 and actin (left). Data represent mean \pm SD from three independent experiments (right). ${ }^{*} \mathrm{P}<0.05$ vs. the control. NSCLC, non-small cell lung cancer; ESE-1, epithelial-specific ETS-1.

using a xenograft model. The LacZ- or ESE-1-expressing H1299 stable cell lines were subcutaneously injected into the flank of nude mice. Fig. 4A shows representative images of the mice of each group. The mice implanted with ESE-1-expressing cells had smaller tumors than those noted in the control mice (Fig. 4A and B). Consistent with these images, the volume and weight of the tumors were significantly decreased in the ESE-1-expressing group compared with the control group (Fig. 4C and D). On day 35, we isolated the tumor tissues and performed immunohistochemistry with antibodies against ESE-1, PCNA and cleaved caspase 3. Expression of ESE-1 in the tumor tissues was associated with a decrease in PCNA (proliferating marker) and an increase in cleaved caspase 3 (Fig. 4E). Western blot analysis indicated that the expression of cleaved caspase- 3 was increased in ESE-1-expressing tumor tissues (Fig. 4F). Taken together, ESE-1 exhibits anticancer activity in vivo, similar to what was observed in vitro.

\section{Discussion}

Due to the fact that ESE-1 is constitutively expressed in terminally differentiated epithelial cells $(7,18)$ and that $80 \%$ of cancer is epithelial origin carcinoma, the significance of this protein was broadly proposed during progression in malignant neoplasms of epithelial origin. However, the effects of ESE-1 on tumorigenesis are controversial depending on the type of cancer (9-11). Therefore, further extensive studies concerning the association of ESE-1 with cancer progression are required using diverse cancer models.

Lung cancer is the most life-threatening cancer due to the difficulty of early detection and poor prognosis. In particular, NSCLC is associated with a poor survival rate due to the acquired resistance of patients to therapeutic drugs. Targeted therapy is a new therapeutic paradigm to cure cancer by targeting specific and promising molecular targets. Recently, Wang et al reported that Elf3 (ESE-1) facilitates the growth 
A

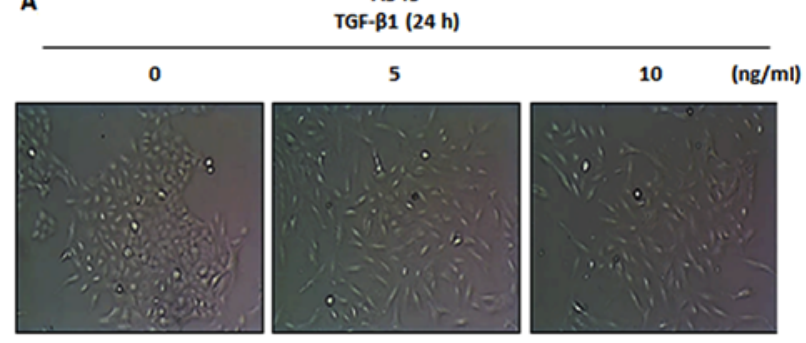

B

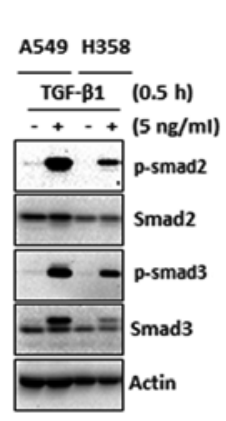

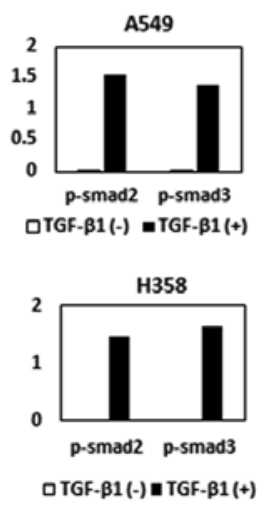

H358

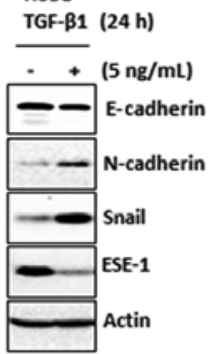

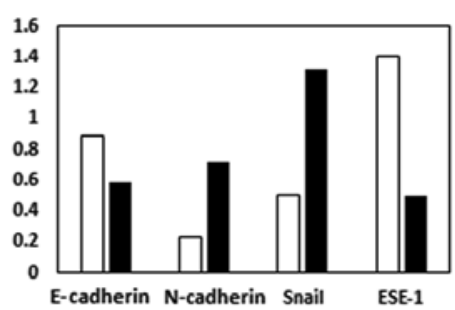

QTGF- $\beta 1(-) \quad=$ TGF- $\beta 1(+)$

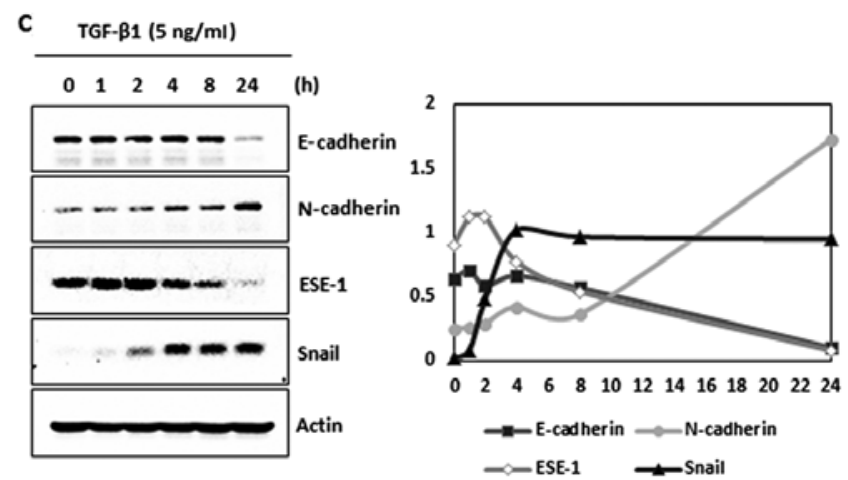

D
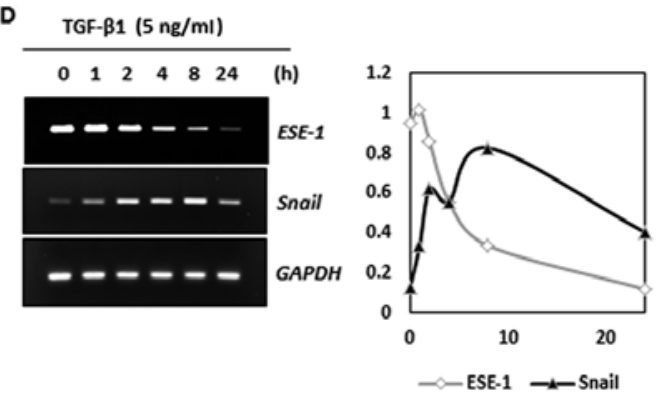

E

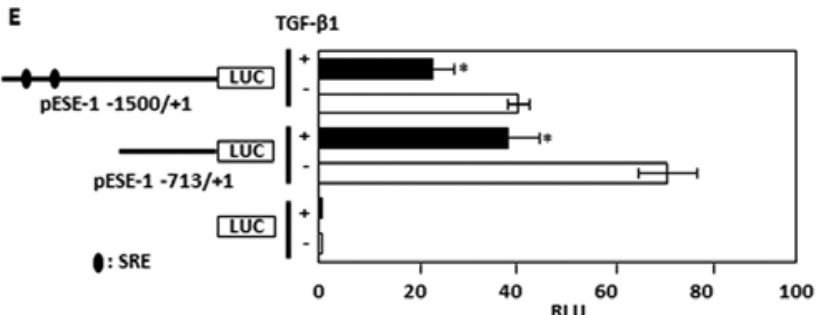

Figure 3. ESE-1 is a target of TGF- $\beta$ during EMT in human NSCLC cells. (A) A549 cells were treated with TGF- $\beta 1(0,5$, and $10 \mathrm{ng} / \mathrm{ml})$ for $24 \mathrm{~h}$. The images were captured by a microscope. (B) A549 and H358 cells were treated with TGF- $\beta 1$ for the indicated doses for 30 min and $24 \mathrm{~h}$. The cell lysates were subjected to western blot analysis for the indicated antibodies. (C) A549 cells were treated with TGF- $\beta 1$ ( $5 \mathrm{ng} / \mathrm{ml})$ for the indicated times. The cell lysates were subjected to western blot analysis for E-cadherin, N-cadherin, ESE-1, Snail and actin. (D) A549 cells were treated with TGF- $\beta 1$ (5 ng/ml) for the indicated times. RNA was isolated and semi-quantitative reverse transcriptase (RT)-PCR was performed for ESE-1, Snail and GAPDH. (E) Two different sizes of the ESE-1 promoter (-1,500/+1 and -173/+1) were cloned as described in Materials and methods and transfected into A549 cells and luciferase activity was measured. Data represent mean \pm SD from three replicates. " $\mathrm{P}<0.05$ vs. the control. NSCLC, non-small cell lung cancer; ESE-1, epithelial-specific ETS-1; TGF- $\beta$, transforming growth factor $\beta$; EMT, epithelial-mesenchymal transition.

and metastasis of lung cancer cells (19). We also explored the possibility of using ESE-1 as a molecular target for lung cancer in the present study.

In the initial step of our studies, we observed endogenous expression of ESE-1 in four different human NSCLC cell lines. As a result, A549 and H358 were identified as ESE-1 abundant cells, whereas H1299 and H1703 were ESE-1 null cells. We did not ascertain why H1299 and H1703 NSCLC cells show very low expression of ESE-1 at the basal level. We speculate that the different basal expression might be a result of genetic/epigenetic difference in the process of $E S E-1$ gene transcription. This is supported by differential 
Lacz

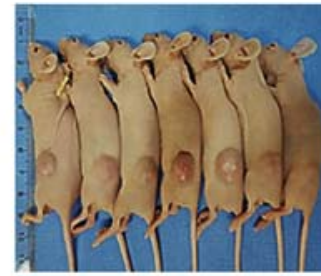

ESE-1

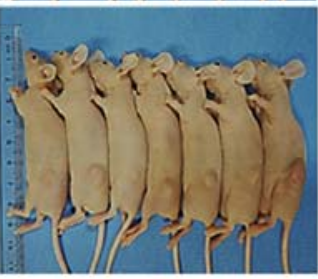

B

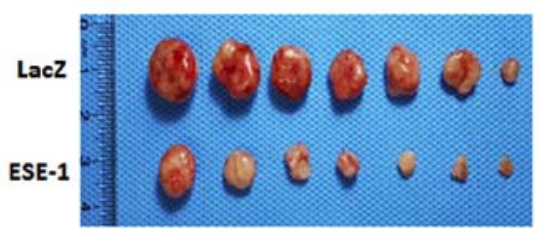

E

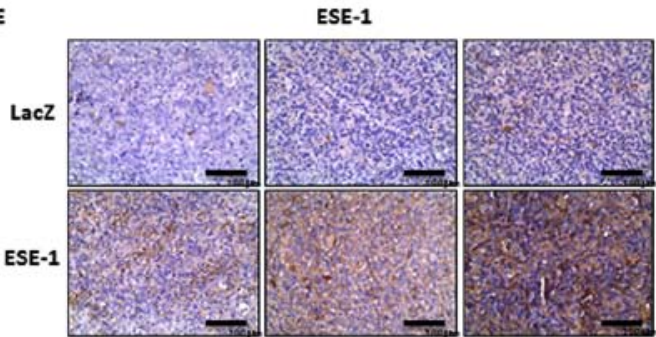

PCNA
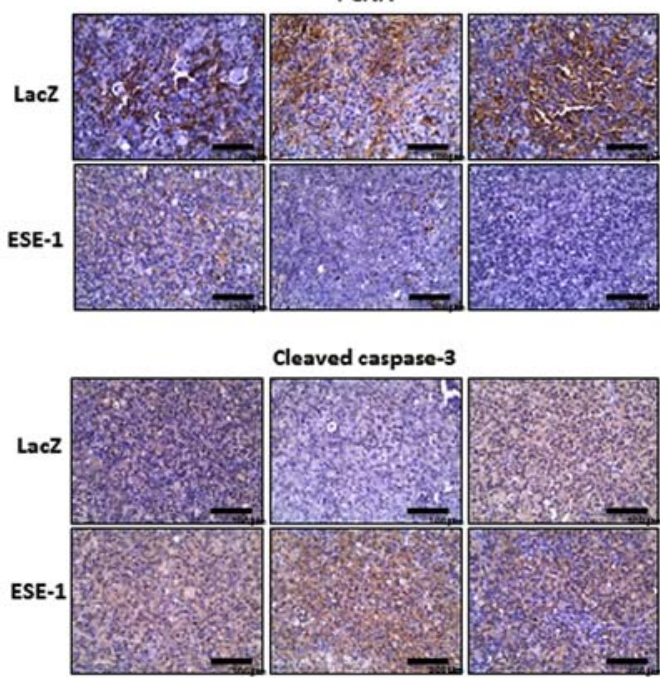

c

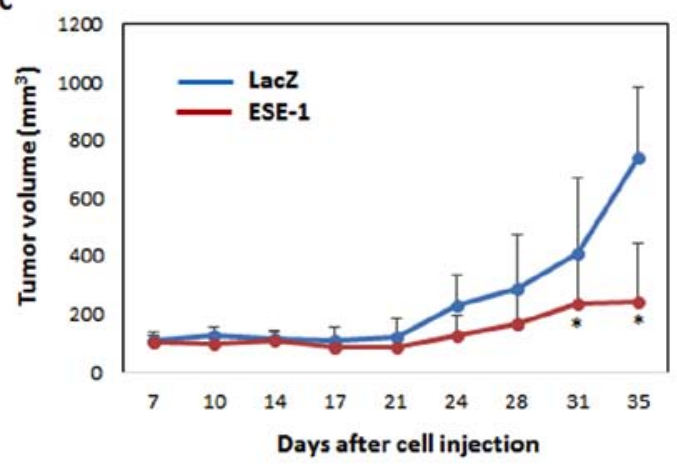

D

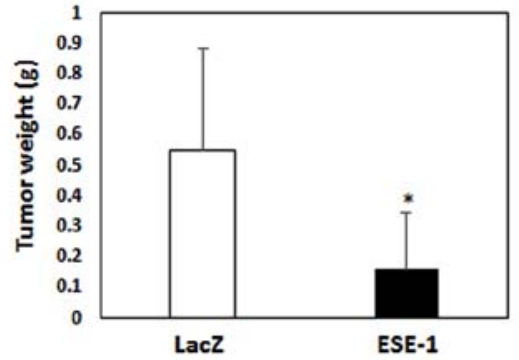

$\mathbf{F}$
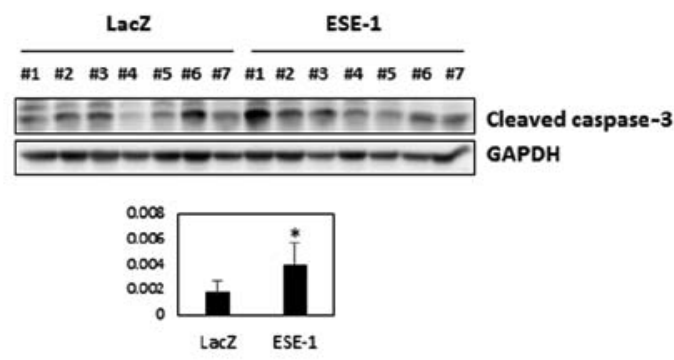

Figure 4. ESE-1 suppresses the formation and development of tumors in vivo. Stable cell lines overexpressing LacZ and ESE-1 were subcutaneously injected into nude mice. On day 35 after injection, the mice were sacrificed. (A) Representative images of xenograft tumors. (B) Comparison of extracted tumors. (C) Mean tumor volume. Data are expressed as mean \pm SD of 7 mice. (D) Mean tumor weight. (E) Immunohistochemistry from excised tumors was performed using antibodies against ESE-1, PCNA and cleaved caspase-3. (F) Western blot analysis was performed for cleaved caspase-3. Quantitative data are represented as mean $\pm \mathrm{SD}$. ${ }^{*} \mathrm{P}<0.05$ vs. the control. ESE-1, epithelial-specific ETS-1.

expression of ESE-1 in different human colorectal cancer cells as we previously reported (20). However, we do not exclude the possibility that there could be differential autocrine regulation of ESE-1 by TGF- $\beta$ among these cell lines 
as lung tumor cells secrete TGF- $\beta$ which may repress ESE-1 expression.

We selected the H1299 cell line for further studies due to its potent tumorigenic and metastatic property (21). Using soft agar assay, we identified ESE-1 as a tumor suppressor in human NSCLC cells. As a part of a mechanistic link, we observed increased G1 arrest and apoptosis in ESE-1-expressing cells which were consistent with the upregulation or downregulation of cell cycle- and apoptosis-regulating proteins (Fig. 1F).

Regarding a role of ESE-1 in metastasis, Yeung et al recently reported that ESE-1 is a negative regulator of EMT and metastasis in ovarian cancer (10). Upregulation of ESE-1 inhibited ovarian tumor progression with increased expression of epithelial markers and decreased expression of mesenchymal markers (10). This is consistent with our observation, supporting the anti-metastatic activity of ESE-1 with suppression of cell invasion and migration and downregulation of MMP-9, VEGF and ZO-1 (Fig. 2).

Epithelial-mesenchymal transition (EMT) is a dynamic process that allows epithelial cells to lose polarity and transit into mesenchymal characteristics. Activation of EMT transcription factors leads to downregulation of E-cadherin (epithelial marker), upregulation of $\mathrm{N}$-cadherin and vimentin (mesenchymal markers), loss of cell adhesion and promotion of cell mobility and invasion (22). TGF- $\beta$ facilitates EMT through the Smad-dependent pathway known as the canonical pathway (23). Besides typical Smad activation, TGF- $\beta$ also induces ERK, JNK/p38, PI3K/AKT, and Rho-like GTPases activation, known as the non-canonical or Smad-independent pathway (24). Several studies support a potential role of ESE-1 in EMT. For example, ESE-1 reciprocally regulates expression of ZEB1/ZEB2, dependent on ERK activity in breast cancer cells (25) and ESE-1 increases expression of TGF- $\beta$ II receptor in breast cancer cells (26).

To elucidate the anti-metastatic mechanism, we focused on the involvement of ESE-1 in EMT of lung cancer cells. Our significant finding was that ESE-1 is a negative downstream target of TGF- $\beta$ signaling. Time-dependent downregulation of ESE-1 was detected in the presence of TGF- $\beta$ (Fig. 3C and D). To investigate if this downregulation is associated with transcriptional repression, we cloned the ESE-1 promoter spanning $-1,500$ to +1 and -713 to +1 . The results indicated that TGF- $\beta$ suppresses transcription of the ESE-1 gene. Since Smads are major mediators of TGF- $\beta$ signaling and we identified two Smad responsive elements (SRE) in the promoter (Fig. 3E), we hypothesized that Smad may mediate TGF- $\beta$-induced downregulation of the ESE-1 gene. However, TGF- $\beta$-induced repression of luciferase activities was identical in the two promoters where one contained SRE and the other did not (Fig. 3E). In addition, luciferase assays using internal deletion and point mutated clones lacking distal and proximal SRE (Table I) showed no difference in the TGF- $\beta$ effect (data not shown). Knockdown of Smad2, Smad3 and Smad4 using siRNA and western blot data also indicated Smad-independent downregulation of ESE-1 by TGF- $\beta$ (data not shown). Therefore, we tested several non-canonical pathways (ERK, p38 MAPK, JNK, RAS, GSK3, PI3K and NF-KB) using selective inhibitors. None of the pathways were responsible for ESE-1 downregulation by TGF- $\beta$ (data not shown).
Concerning the potential mechanism by which ESE-1 suppresses lung cancer progression, we propose several cancer-related downstream target genes of ESE-1. One of the targets is MMP-9 which was downregulated by ESE-1 through ETS-binding site on the MMP-9 promoter in squamous cell carcinoma (11). ESE-1 downregulates OCT4, a transcription factor, involved in stem cell pluripotency of NCCIT human embryonic carcinoma cells (27). Another interesting target gene we propose is wnt $/ \beta$-catenin as we previously observed that ESE- 1 physically interacts with $\beta$-catenin (20) and suppresses transcriptional activity of $\beta$-catenin/LEF (data not shown). Since $\beta$-catenin and APC mutations are uncommon in NSCLC, this mechanism could be promising to elucidate the mechanism of ESE-1 in regard to the suppression of lung cancer progression. Therefore, further research is warranted to elucidate further detailed mechanisms of ESE-1 downregulation by TGF- $\beta$ and molecular target mediating anticancer activity of ESE-1 in human NSCLC cells.

In conclusion, we identified ESE-1 as a tumor suppressor in human NSCLC. ESE-1 represses anchorage-independent growth and invasion and migration of human NSCLC cells. ESE-1 is a target of TGF- $\beta$ during EMT. In addition, ESE-1 suppressed the formation and development of tumors in vivo.

\section{Acknowledgements}

The authors thank Jihye Lee for her technical supports.

\section{Funding}

The present study was supported by a grant from the National Research Foundation of Korea (SRC, no. 2011-0030043: no. 2018R1A2B3009008).

\section{Availability of data and materials}

The datasets used during the present study are available from the corresponding author upon reasonable request.

\section{Authors' contributions}

SHL and CHK conceived and designed the study. ZL, BSL, TH, YX and HJK performed the experiments. ZL, BSL and TH wrote the paper. SHL reviewed and edited the manuscript. All authors read and approved the manuscript and agree to be accountable for all aspects of the research in ensuring that the accuracy or integrity of any part of the work are appropriately investigated and resolved.

\section{Ethics approval and consent to participate}

All experimental protocols were approved by the Animal Care and Use Committee (Ajou University, Korea).

\section{Patient consent for publication}

Not applicable.

\section{Competing interests}

The authors state that they have no competing interests. 


\section{References}

1. Siegel RL, Miller KD and Jemal A: Cancer statistics, 2017. CA Cancer J Clin 67: 7-30, 2017.

2. Schiller JH, Gandara DR, Goss GD and Vokes EE: Non-small-cell lung cancer: Then and now. J Clin Oncol 31: 981-983, 2013. doi: $10.1200 / J C O .2012 .47 .5772$.

3. Tymms MJ, Ng AY, Thomas RS, Schutte BC, Zhou J, Eyre HJ, Sutherland GR, Seth A, Rosenberg M, Papas T, et al: A novel epithelial-expressed ETS gene, ELF3: Human and murine cDNA sequences, murine genomic organization, human mapping to 1q32.2 and expression in tissues and cancer. Oncogene 15 : 2449-2462, 1997.

4. Choi SG, Yi Y, Kim YS, Kato M, Chang J, Chung HW, Hahm KB, Yang HK, Rhee HH, Bang YJ and Kim SJ: A novel ets-related transcription factor, ERT/ESX/ESE-1, regulates expression of the transforming growth factor- $\beta$ type II receptor. J Biol Chem 273: 110-117, 1998.

5. Chang CH, Scott GK, Kuo WL, Xiong X, Suzdaltseva Y, Park JW, Sayre P, Erny K, Collins C, Gray JW, et al: ESX: A structurally unique Ets overexpressed early during human breast tumorigenesis. Oncogene 14: 1617-1622, 1997.

6. Oettgen P, Finger E, Sun Z, Akbarali Y, Thamrongsak U, Boltax J, Grall F, Dube A, Weiss A, Brown L, et al: PDEF, a novel prostate epithelium-specific ets transcription factor, interacts with the androgen receptor and activates prostate-specific antigen gene expression. J Biol Chem 275: 1216-1225, 2000.

7. Oettgen P, Alani RM, Barcinski MA, Brown L, Akbarali Y, Boltax J, Kunsch C, Munger K and Libermann TA: Isolation and characterization of a novel epithelium-specific transcription factor, ESE-1, a member of the ets family. Mol Cell Biol 17: 4419-4433, 1997.

8. Brembeck FH, Opitz OG, Libermann TA and Rustgi AK: Dual function of the epithelial specific ets transcription factor, ELF3, in modulating differentiation. Oncogene 19: 1941-1949, 2000.

9. Wang JL, Chen ZF, Chen HM, Wang MY, Kong X, Wang YC, Sun TT, Hong J, Zou W, Xu J, et al: Elf3 drives $\beta$-catenin transactivation and associates with poor prognosis in colorectal cancer. Cell Death Dis 5: e1263, 2014.

10. Yeung TL, Leung CS, Wong KK, Gutierrez-Hartmann A, Kwong J, Gershenson DM and Mok SC: ELF3 is a negative regulator of epithelial-mesenchymal transition in ovarian cancer cells. Oncotarget 8: 16951-16963, 2017.

11. Iwai S, Amekawa S, Yomogida K, Sumi T, Nakazawa M, Yura Y, Nishimune Y and Nozaki M: ESE-1 inhibits the invasion of oral squamous cell carcinoma in conjunction with MMP-9 suppression. Oral Dis 14: 144-149, 2008.

12. Lee SH, Bahn JH, Choi CK, Whitlock NC, English AE, Safe S and Baek SJ: ESE-1/EGR-1 pathway plays a role in tolfenamic acid-induced apoptosis in colorectal cancer cells. Mol Cancer Ther 7: 3739-3750, 2008.

13. Longoni N, Sarti M, Albino D, Civenni G, Malek A, Ortelli E, Pinton S, Mello-Grand M, Ostano P, D'Ambrosio G, et al: ETS transcription factor ESE1/ELF3 orchestrates a positive feedback loop that constitutively activates NF-kappaB and drives prostate cancer progression. Cancer Res 73: 4533-4547, 2013.
14. Shatnawi A, Norris JD, Chaveroux C, Jasper JS, Sherk AB, McDonnell DP and Giguère V: ELF3 is a repressor of androgen receptor action in prostate cancer cells. Oncogene 33: 862-871, 2014.

15. Oliver JR, Kushwah R, Wu J, Pan J, Cutz E, Yeger H, Waddell TK and $\mathrm{Hu}$ J: Elf3 plays a role in regulating bronchiolar epithelial repair kinetics following Clara cell-specific injury. Lab Invest 91: 1514-1529, 2011.

16. Lee SH, Bahn JH, Whitlock NC and Baek SJ: Activating transcription factor 2 (ATF2) controls tolfenamic acid-induced ATF3 expression via MAP kinase pathways. Oncogene 29: 5182-5192, 2010.

17. Lee SH, Cekanova M and Baek SJ: Multiple mechanisms are involved in 6-gingerol-induced cell growth arrest and apoptosis in human colorectal cancer cells. Mol Carcinog 47: 197-208, 2008.

18. Ng AY, Waring P, Ristevski S, Wang C, Wilson T, Pritchard M, Hertzog P and Kola I: Inactivation of the transcription factor Elf3 in mice results in dysmorphogenesis and altered differentiation of intestinal epithelium. Gastroenterology 122: 1455-1466, 2002.

19. Wang H, Yu Z, Huo S, Chen Z, Ou Z, Mai J, Ding S and Zhang J: Overexpression of ELF3 facilitates cell growth and metastasis through PI3K/Akt and ERK signaling pathways in non-small cell lung cancer. Int J Biochem Cell Biol 94: 98-106, 2018.

20. Yang $X$ and Lee SH: Identification of ESE1 as a $\beta$-Catenin Binding Protein. Anticancer Res 36: 2697-2703, 2016.

21. Singla AK, Downey CM, Bebb GD and Jirik FR: Characterization of a murine model of metastatic human non-small cell lung cancer and effect of CXCR4 inhibition on the growth of metastases. Oncoscience 2: 263-271, 2015.

22. Thiery JP and Sleeman JP: Complex networks orchestrate epithelial-mesenchymal transitions. Nat Rev Mol Cell Biol 7: 131-142, 2006.

23. Derynck R and Zhang YE: Smad-dependent and Smad-independent pathways in TGF-beta family signalling. Nature 425: 577-584, 2003.

24. Zhang YE: Non-Smad pathways in TGF-beta signaling. Cell Res 19: 128-139, 2009.

25. Sinh ND, Endo K, Miyazawa K and Saitoh M: Ets1 and ESE1 reciprocally regulate expression of ZEB1/ZEB2, dependent on ERK1/2 activity, in breast cancer cells. Cancer Sci 108: 952-960, 2017.

26. Chang J, Lee C, Hahm KB, Yi Y, Choi SG and Kim SJ: Overexpression of ERT(ESX/ESE-1/ELF3), an ets-related transcription factor, induces endogenous TGF-beta type II receptor expression and restores the TGF-beta signaling pathway in Hs578t human breast cancer cells. Oncogene 19: 151-154, 2000.

27. Park SW, Do HJ, Ha WT, Han MH, Yang HM, Lee SH, Song H, Kim NH and Kim JH: Transcriptional regulation of OCT4 by the ETS transcription factor ESE-1 in NCCIT human embryonic carcinoma cells. Biochem Biophys Res Commun 450: 984-990, 2014. 